\title{
Étude électrophorétique des protéines sériques d'herbivores et d'oiseaux de la faune tchadienne. Premiers résultats
}

\author{
por R. QUEVAL (*) \\ (avec la collaboration de Madame M. BROCK)
}

\begin{abstract}
RÉSUMÉ
L'analyse électrophorétique des constıtuants protidiques du sérum d'animaux sauvages et de leur mobilité relative a été réalısée sur 26 genres zoologiques comprenant 119 sérums de mammifères et 74 sérums d'oiseaux appartenant à la faune cynégétique tchadienne.
\end{abstract}

\section{INTRODUCTION}

La technique d'électrophorèse sur papier est devenue aujourd'hui une méthode sımple et rapide, permettant l'isolement des fractions sériques et leur étude subséquente après un fractionnement physique de leurs molécules protéiniques ionisées.

Ce mode d'investigation est largement utılisé en médecine humaine : d'une part c'est une méthode d'estimation quantitative permettant de définir la proportion relative de chaque constituant individualisable à l'intérieur des différents composants protéiniques (albumines et globulines), d'autre part c'est une méthode de diagnostic commode dans certaines affecticns (néphrose lıpoïdique, myélome multiple, hémoglobinoses, atteintes hépatiques, etc.).

En médecine vétérinaire, la méthode électrophorétique s'est vulgarisée plus tardivement à

(*) I.E.M.V.T. laboratoire de ı echerches vétérinaires de Farcha, Fort-Lamy, Tchad. l'étude comparative des sérums normaux ef pathologiques des animaux domestiques ef de laboratoire.

Les valeurs normales et les variations physiologiques des protéines sériques désormais bien établies chez les animaux domestiques sont rares chez les anımaux sauvages.

Le but du présent travail n'est pas de définir le protéinogramme type du sérum des espècessauvages (mammifères ou oiseaux) ! ce mode d'investigation électrophorétique nécessite un grand nombre d'observations. Plusieurs nombres de paramètres doivent en outre être précisés (conditions physiologiques, pathologiques, écologiques).

Cependant, une telle étude semble intéressante et se doit d'être abordée pour contribuer à l'établissement des constantes électrophorétiques de chaque espèce sauvage qui permettront une comparaison entre elles, de même qu'avec celles. de I'homme et des animaux domestiques. En. outre, elles seront un point de départ pour les recherches de physiologie animale comparée. 


\section{MATÉRIEL ET MÉTHODE}

\section{Espèces ef aires géographiques.}

Les observations ont porté sur sept ordres zoologiques : artiodactyles, proboscidiens, carnivores et insectivores pour les mammifères; ansériformes, gruiformes et ardéiformes pour les oiseaux, comprenant 26 genres différents : phacochère (Phacochoerus aethiopicus, Pallas) ; hippopotame (Hippopotamus amphibrus L.) ; buffle (Syncerus coffer aequinoxiolis, Blyth) : bubale rouge (Alcelaphus lelwel, Heuglin); damalisque (Damoliscus korrigum, Ogilby) : cephalophe (Sylvicapra grımmia, Linné) ; ourebi à front noir (Ourebia ourebi dorcas, Schw.) cob de Buffon (Adenoto kob, Erxleben) ; cob onctueux (Kobus defessa, Rüppel) ; gazelle des sables (Gazella dorcas dorcas, Linné) ; Gazelle corinne (Gozella rufifrons, Gray) ; biche Robert (Gozella dama, Pallas) ; hippotrague ou Antilope cheval (Hippotragus equinus, Desmaret) ; Oryx (Oryx Aegorix algazel, Oken) ; élan de Derby (Tourotragus derbionus, Gray) ; éléphant (Loxodonta africana, Blumenbach); mangouste (Ichneumia albicauda, Cuvier) ; hérisson Atelerix (Erinoceus) albiventris spiculus, Thomas et Wroughton).

Parmi les oiseaux : marabout (Leptoptilos crumeniferus, Lesson); oie armée (Plectropterus gambensis, Linné) ; oie caronculée (Sarkidiornis melanotos, Pennant); oie d'Egypte (Alopochen aegyptiacus, Linné) ; canard siffleur (Dendrocygna viduata, Linné); pintade (Numida meleagris, Linné) ; outarde (Choriotis arabs Stieberi, Neumann).

Au total, les constituants protidiques du sérum des animaux sauvages ont été analysés sur 198 sérums dont 119 appartiennent aux mammifères et 79 aux oiseaux.

Les anımaux ont été soit abattus au titre d'un permis de chasse scientifique, soit capturés et destinés à l'exportation.

Les lieux de chasse et de capture dans les zones cynégétiques tchadiennes sont : le Nord du Ouaddaï et la préfecture de Biltine ; la bande du $14 \mathrm{e}$ paralièle entre Mao et Moussoro ainsi que le Nord-Est de cette préfecture, enfin la région située de part et d'autre du Chari autour de l'intersection du $10^{\mathrm{e}}$ parallèle et du $18^{\mathrm{e}}$ degré de longitude.

\section{Prélèvements.}

Lors de safaris, le sang est récolté immédiatement après l'abattage ; lorsqu'il s'agit d'animaux capturés, les prélèvements sanguins sont réalisés par voie intraveineuse.

Bien qu'ils aient été effectués obligatoirement dans des lieux très éloignés du laboratoire, malgré ces circonstances, après coagulation et rétraction, la séparation du caillot-sérum a pu être rapidement effectuée de manière à mettre un terme aux échanges s'effectuant, in vitro, entre ces deux parties du sang.

Les échantillons de sérum sont placés dans la glace fondante pour leur transportau laboratoire et ensuite conservés au congélateur d̀ To $-20^{\circ}$.

\section{Méthode.}

L'analyse électrophorétique des sérums est réalısée sur l'appareil horizontal de WIELAND et FISHER.

Le tampon véronal de Durrum de $\mathrm{pH} 8,6$ et de force ionique 0,05 des bandes de papier Arches 304 et une alimentation en haute tension continue de 400 volts pendant 210 minutes sont les paramètres constants des conditions expérimentales.

Après séparation électrophorétique, les bandes de papier sont séchées à l'infra-rouge et les protéines révélées par coloration au bleu de bromophénol.

L'appareil permet l'enregistrement photométrique par transparence et transcription en densités optiques. La courbe photométrique est intégrée pour calculer la valeur relative, en pourcentage, de chacune des fractions électrophorétiques.

Sımultanément, les protéines totales et l'ałbumine sont dosées par la méthode colorimétrique au biuret, selon la technique de GORNALL et BARDAWILL (1949) modifiée par FLEURY et EBERHARDT (1951).

L'appareil utilisé est le protocolorımètre Lumétron; les mesures photométriques sont réalisées en lumière monochromatique à 540 millimicrons.

\section{RÉSULTATS}

\section{Qualitatifs.}

Les résultats qualitatıfs et quantitatifs ont été obtenus par l'analyse du spectre de migration 
sur papier des fractions protéiniques et du diagramme d'enregistrement photométrique de la bande colorée ainsi que de l'intégration de la courbe d'opacité.

Dans l'interprétation des résultats, la première des diffıcultés rencontrées est d'ordre terminologique. En effet, les principales fractions protéiniques sont définies selon leur vitesse de migration électrophorétique et ce critère est valable pour tous les sérums d'où une nomenclature commune aux constituants des sérums humains et animaux, bien qu'il n'y ait pas une identité chimique absolve.

Quant aux sous-fractions des principaux constituants, leur nature et leur nombre varient largement d'une espèce à l'autre et leurs appellations n'offrent qu'une correspondance arbitraire.

\subsection{L'Electroproteinogramme.}

Après séparation, fixation et coloration, le simple examen des bandes de papier montre des zones plus ou moins individualisées et de coloration d'intensité inégale qui correspondent aux fractions protéiniques de mobilité différente.

Ce diagramme d'étalement met en évidence des différences notables dans le nombre des fractions protéiques révélées par l'électrophorèse sur papier.

Chez les mammifères, seul le hérisson présente 5 bandes distinctes, soit 1,6 p. 100 des sérums. Les tracés de ce type sont similaires au protéinogramme classique du sérum humain où l'on distingue entre le point de départ de migration du sérum et la tache nette à l'extrémité opposée du diagramme, correspondant à la fraction albumine, les fractions suivantes : par ordre de mobilités décroissantes : alpha 1 globulines, alpha 2 globulines, beta globulines et gamma globulines, fraction la plus lente et sise près de l'origine.

Chez les oiseaux, aucun diagramme de ce type n'a été rencontré.

Pour 43 p. 100 des sérums de mammifères et 40,6 p. 100 des sérums d'oiseaux, le protéınogramme ne présente que 4 bandes distinctes représentant les albumines et les globulınes alpha, beta et gamma.

L'examen du diagramme protéinique, sans photométrie, révèle encore pour 47,8 p. 100 des sérums de mammifères et pour 36,7 p. 100 des sérums d'oiseaux un protéınogramme où l'on ne distingue plus que 3 taches neftement séparées.

La tache la plus éloignée de l'origine (la sérum albumine) et celle située près du point de départ (la gamma globulıne) ont un aspect homogène et sont uniformément colorées. Entre ces deux fractions, la troisième tache présente des zones de coloration d'intensıté inégale où l'on ne peut discerner sans avorr recours à la photométrie, les fractions alpha et beta globuline qui constıtuent cette troisıème tache.

Enfin, dans 7,6 p. 100 et 22,7 p. 100 des cas respectivement pour les sérums de mammıfères et d'oiseaux, le diagramme électrophorétique ne présente plus que 2 bandes distinctes: une tache homogène de coloration uniforme, bien indıvidualisée: l'albumıne, ef une seconde bande hétérogène avec des gradients de coloration qui figurent les fractions globuliniques (alpho, beta, et gamma globulines).

\subsection{La courbe d'enregistrement photométrique.}

La lecture photométrique offre une analyse plus fine du protéinogramme, la courbe enregistrée présentant des accidents tels que pics, vallées et inflexions qui correspondent exactement aux divers gradients de coloration du protéinogramme. Une simple juxtaposition du protéinogramme et de la courbe photométrique permet l'identification des principales fractions électrophorétiques. En effet, la complexité de certaines courbes, due aux proportions variables et aux écarts individuels de mobilité de certaines fractions, interdit la distinction de sous-fractions, voire de fractions.

Dans bien des cas l'identification des courbes de Gauss correspondant aux sous-fractions (alpha 1 et 2, beta 1 et 2, et gamma 1 et 2) n'est pas suffisamment nette : pour être à l'abri d'interprétations subjectives, on s'est efforcé, outre les albumines, à ne différencier que les globulines alpha, beta et gamma.

Ont été éliminés également les sérums pour lesquels la différenciation des fractions se limite uniquement soit aux albumines ef aux globulines, solf à l'ındividualisatıon de la sérum albumine, de la totalité des globulines alpha et beta, et des gamma globulines.

Pour chaque protéinogramme ont été déter- 
minées les positions relatives des différentes fractions protéiques selon le procédé de DREVON (1953). II consiste à mesurer les distances qui séparent les pieds des perpendiculaires abaissées des sommets des pics sur la ligne de base. Ces distances mesurées au double décimètre, une simple règle de trois permet de les rapporter à la distance arbitraırement notée égale d̀ 100 millimètres entre le point d'origine de migration ef l'album!ne,

Cette méthode permet une représentation graphique lorsque les sondages portent sur des effectifs importants. En outre, elle est très utile dans l'identification d'une fraction sur un tracé complexe.

Ces mobilités relatives des diverses fractions électrophorétiques de diverses espèces animales sont rassemblées dans les tableaux 1 et 2 . La moyenne arithmétique représente la distance en millimètres d̀ partir du point d'origine, les tracés étant rapportés à $100 \mathrm{~mm}$. A côté de la moyenne arithmétique figure son écart standard.

TABLEAU $\mathrm{N}^{\circ} \mathrm{I}$

Distances en millimètres à partir du point de départ de la migration électrophorétique. Mammifères.

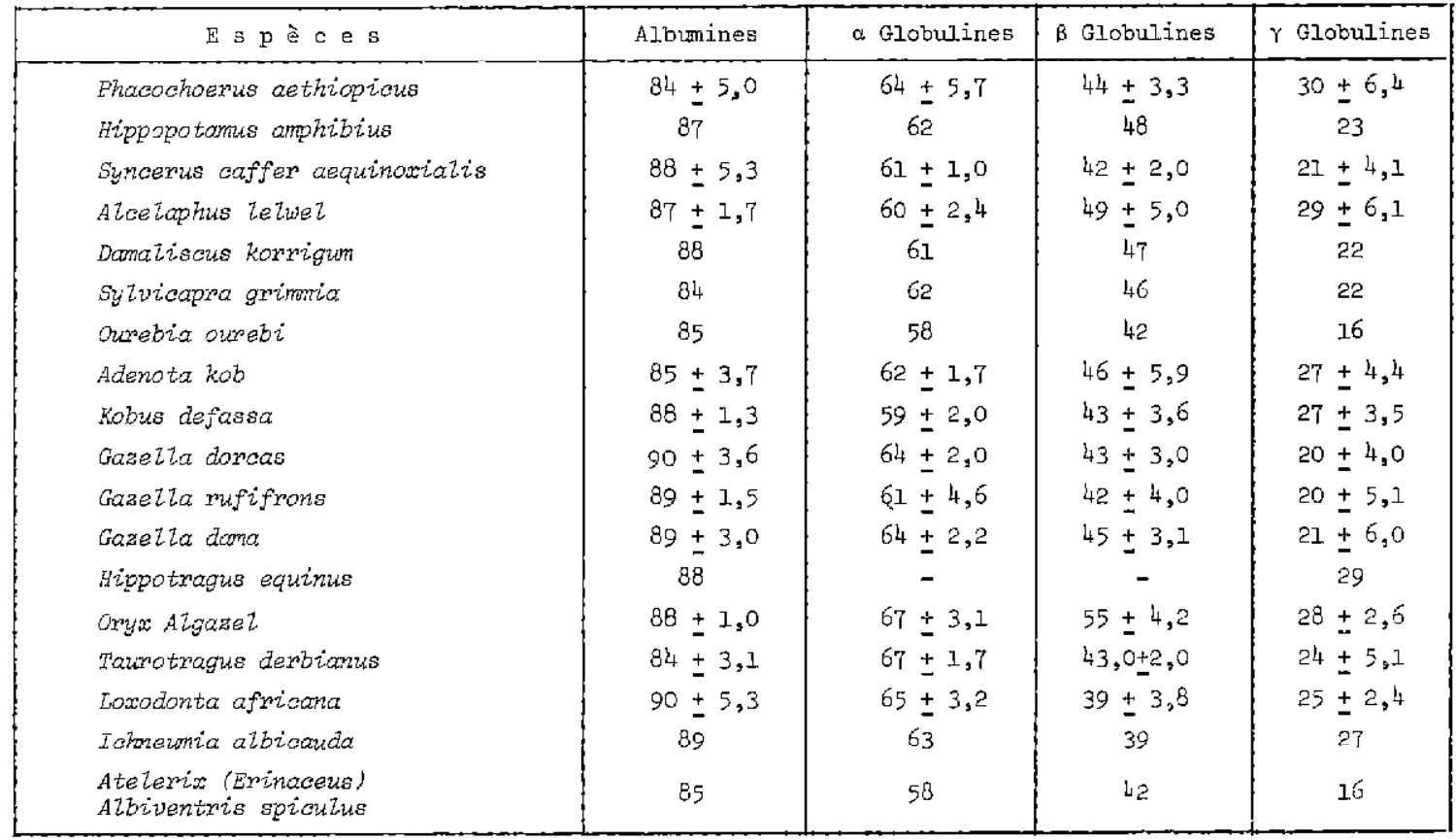

TABLEAU $\mathrm{N}^{\circ}$ II

Distances en millimètres à partir du point de départ de la migration électrophorétıque - Oıseaux.

\begin{tabular}{|c|c|c|c|c|c|c|}
\hline Es $\mathrm{p}$ è $c \in \mathrm{s}$ & Albumines & $\alpha$ & Globulines & $\beta$ & Globulines & $\gamma$ Globulines \\
\hline $\begin{array}{l}\text { Plectroptemus gambensis } \\
\text { Samkidionis melanotos } \\
\text { Azopochen aeguptiacus } \\
\text { Dendrocygna viduata } \\
\text { Numida meteagmis } \\
\text { Chomiotis arabs }\end{array}$ & $\begin{array}{c}79 \pm 5,5 \\
84 \\
85 \pm 4,3 \\
83 \pm 3,3 \\
85 \pm 4,1 \\
=90\end{array}$ & & $\begin{array}{l}57 \pm 5,3 \\
59 \\
60 \pm 4,1 \\
53 \pm 1,4 \\
53 \pm 5,0 \\
=62\end{array}$ & & $\begin{array}{l}4 I \pm 5,4 \\
41 \\
43 \pm 3,7 \\
38 \pm 2,8 \\
-46 \pm \\
\pm 4,0 \\
34\end{array}$ & $\begin{array}{l}31 \pm 5,0 \\
26 \\
32 \pm 5,4 \\
28 \pm 1,7 \\
27 \pm 3,0 \\
\pm \\
18\end{array}$ \\
\hline
\end{tabular}


L'étude du tracé photométrique permet de reconnaître au sein de certaınes fractions deux composants. Par exemple, la fraction a globuline présente 2 sommets chez l'élan de Derby, situés d'après les mobilités relatives, à 58 et $67 \mathrm{~mm}$, et chez le canard siffleur à 53 et $61 \mathrm{~mm}$.

La fraction beta globulıne se scinde en deux composants chez l'éléphant (51 et $39 \mathrm{~mm}$ ), l'élan de Derby ( 49 et $43 \mathrm{~mm}$ ) ef le phacochère (53 et $44 \mathrm{~mm}$ ).

La fraction gamma globulinique révèle deux sommets situés à 53 et $24 \mathrm{~mm}$ chez l'élan de Derby et à 32 et $16 \mathrm{~mm}$ chez le hérisson.
D'une manière générale, ce procédé d'analyse montre pour les sérums de mammifères et d'oiseaux que les pics correspondant aux diverses fractions (albumines, globuline alpha, beta ef gamma) se groupent, en fonction des mobilités relatives de ces composants, à l'intérieur d'intervalles caractéristiques ainsi que le montre le tableau 3 où figure également la position moyenne.

\section{Résultats quantitatifs.}

Les résultats quantitatifs sont donnés dans les tableaux 4 et 5 où sont présentés la valeur

\section{TABLEAU $\mathrm{N}^{\circ}$ III}

Distances en millimètres à partir du point de départ de la migration électrophorétique.

\begin{tabular}{|c|c|c|c|c|}
\hline Espèces & Albumines & a Globurines & $\beta$ Globulines & $\gamma$ Globulines \\
\hline Manmifères & $95-79 \mathrm{~mm}$ & $70-58 \mathrm{~mm}$ & $59-35 \mathrm{~mm}$ & $36-15 \mathrm{~mm}$ \\
& $87 \mathrm{~mm}$ & $62 \mathrm{~mm}$ & $44 \mathrm{~mm}$ & $23 \mathrm{~mm}$ \\
Oi.jeaux & $90-74 \mathrm{~mm}$ & $64-48 \mathrm{~mm}$ & $46-32 \mathrm{~mm}$ & $37-18 \mathrm{~mm}$ \\
& $84 \mathrm{~mm}$ & $57 \mathrm{~mm}$ & $38 \mathrm{rm}$ & $27 \mathrm{Jm}$ \\
\hline
\end{tabular}

TABLEAU $N^{\circ}$ IV

Proportions relatives des fractions protéiques de quelques aninaux saurages.

\begin{tabular}{|c|c|c|c|c|c|}
\hline$E s p e ̀ c e s$ & $\begin{array}{c}\text { Nombre } \\
\text { a'enimaux }\end{array}$ & $\begin{array}{l}\text { Albumines } \\
\text { p. } 100\end{array}$ & $\begin{array}{l}\alpha \text { Globulines } \\
\text { p.100 }\end{array}$ & $\begin{array}{c}\text { B Globulines } \\
\text { p. } 100\end{array}$ & $\begin{array}{c}\gamma \text { Globulines } \\
\text { p.100 }\end{array}$ \\
\hline Phacochoerus aethiopicus & 10 & $32,8 \pm 40$ & $21,2 \pm 2,2$ & $17,5 \pm 2,2$ & $28,5 \pm 3,0$ \\
\hline Hippopotomus amphibius & 2 & 32,0 & 10,0 & 17,1 & 40,9 \\
\hline Syncerus caffer aequinoxialic & 5 & $34,0 \pm 1,7$ & $12,3 \pm 0,9$ & $19,4 \pm 2,2$ & $34,3 \pm 3,7$ \\
\hline Alcelophus leiwe $\vec{i}$ & 10 & $35,4 \pm 1,8$ & $12,7 \pm 1,7$ & $16,2 \pm 2,2$ & $35,7 \pm 3,7$ \\
\hline Domaliscus korrigum & 3 & 35,0 & 12,9 & 19,3 & 32,7 \\
\hline Sylviacora grimmia & 2 & 31,7 & 16,6 & 19,9 & 31,7 \\
\hline ourebia ourebi & 1 & 35,3 & 12,8 & 15,7 & 35,7 \\
\hline Adenota kob & 8 & $33,0 \pm 3,5$ & $15,0 \pm 2,0$ & $17,6 \pm 0,28$ & $34,4 \pm 4,0$ \\
\hline Kobus defassa & 6 & $35,8 \pm 2,8$ & $24,6 \pm 1,5$ & $16,8 \pm 1,2$ & $32,8 \pm 1,3$ \\
\hline Redronca arundinum & 4 & 31,3 & 15,6 & 19,2 & 33,8 \\
\hline Gazelza doreas & 7 & $32,3 \pm 2,0$ & $12,5 \pm 1,7$ & $22,7 \pm 3,4$ & $32,4 \pm 3,6$ \\
\hline Gazelza rufifrons & 9 & $32,5 \pm 3,9$ & $15,1 \pm 2,4$ & $22,9 \pm 1,2$ & $29,4 \pm 3,4$ \\
\hline Gazeila dama & $i \in$ & $31,9 \pm 3,4$ & $13,3 \pm 2,1$ & $20,2 \pm 2,6$ & $34,5 \pm 3,0$ \\
\hline Hippotragus é quinus & I & 35,7 & - & - & - \\
\hline arys Algazez & 8 & $35,0 \pm 3,3$ & $12,4 \pm 2,8$ & $21,9 \pm 1,8$ & $30,7 \pm 2,8$ \\
\hline Taurotragus derbionus & 19 & $33,8 \pm 4,1$ & $12,8 \pm 2,0$ & $18,1 \pm 3,6$ & $35,2 \pm 3,9$ \\
\hline Loxodonta africana & 4 & $29,7 \pm 1,7$ & $11,2 \pm 1,4$ & $11,8 \pm 1,6$ & $47,3 \pm 1,8$ \\
\hline Ichneumia albicauda & 2 & 34,2 & 15,3 & 17,3 & 33,1 \\
\hline $\begin{array}{l}\text { Atelerix (Erinaceus) } \\
\text { Albiventis spiculus }\end{array}$ & 2 & 32,4 & 16,2 & 14,5 & $\begin{array}{c}36,8 \\
(13,1+23,7)\end{array}$ \\
\hline
\end{tabular}


Proportions relatives des fractions proteiques de gueloues oiseaux-gibier.

\begin{tabular}{|c|c|c|c|c|c|}
\hline Esp èces & $\begin{array}{l}\text { Nombre } \\
\text { anImaux }\end{array}$ & $\begin{array}{l}\text { Albumines } \\
\text { p. } 100\end{array}$ & $\begin{array}{c}\text { a. Globulines } \\
\text { p.100 }\end{array}$ & $\begin{array}{c}\text { B Globulines } \\
\text { p.100 }\end{array}$ & $\begin{array}{c}\text { Globulines } \\
\text { p. } 100\end{array}$ \\
\hline Leptoptitos crumeniferus & 1 & 29,0 & 25,1 & 10,0 & 35,9 \\
\hline Plectropterus gambensis & 7 & $37,3 \pm 2,0$ & $1 \varepsilon, 8 \pm 2,8$ & $13,2 \pm 2,7$ & $36,7 \pm 2,7$ \\
\hline Sarkidionis meianotos & 2 & 40,0 & 15,0 & 13,0 & 32,0 \\
\hline Atopochen deguttiacus & 25 & $38,8 \pm 4,3$ & $14,0 \pm 2,6$ & $15,1 \pm 2,7$ & $31,3 \pm 4,1$ \\
\hline Dendrocugna viduata & 23 & $35,3 \pm 3,3$ & $18,7 \pm 2,0$ & $14,3 \pm 3,8$ & $31,6 \pm 3,7$ \\
\hline Wrmida me Zeagris & 18 & $35,5 \pm 3,4$ & $19,1 \pm 2,8$ & $12,8 \pm 2,5$ & $32,6 \pm 3,7$ \\
\hline Choriotis arabs & 2 & 35,6 & $1\}, 3$ & 15,3 & 31,8 \\
\hline Accip ter sp. & 1 & 25,6 & 21,2 & 11,4 & 21,8 \\
\hline
\end{tabular}

moyenne des différents constituants électrophorétiques exprimée par leurs proportions relatives en pourcentage ef leurs écarts types correspondants.

L'examen des tableaux montre que les pourcentages des diverses fractions protéiques du sérum des animaux examinés paraissent sensiblement homogènes, constants, dans une même espèce animale et très voisines d'une espèce à l'autre.

Pour les espèces appartenant à la même famille ou sous-famille zoologique, il ne paraît pas y avoir de différence flagrante entre les espèces domestiques et sauvages telles que porc et phacochère, bœuf et buffle, canards domestiques et sauvages.

TABLEAU $\mathbb{N}^{\circ}$ VI

Valeurs absolues des composants proteiniques. (Poids en gramme pour $100 \mathrm{ml}$ de Sêrum sanguin)

\begin{tabular}{|c|c|c|c|}
\hline Es p èces & $\begin{array}{c}\text { Protéines } \\
\text { totales }\end{array}$ & Albumines & Globulines \\
\hline $\begin{array}{l}\quad \text { Memmifères } \\
\text { Phacochoerus aethiopious } \\
\text { Syncems caffer aequinoxialis } \\
\text { Alaelaphus lelwel } \\
\text { Adenota kob } \\
\text { Kobus defassa } \\
\text { Redunca arundinum } \\
\text { Gazella dorcas } \\
\text { Gazelta rufifrons } \\
\text { Gazelta dama } \\
\text { Oryx Algazel } \\
\text { Taurotragus derbianus } \\
\text { Loxodonta africana }\end{array}$ & $\begin{array}{l}7,5 \\
6,75 \\
6,73 \\
9,0 \\
7,85 \\
5,89 \\
5,44 \\
6,33 \\
6,34 \\
5,91 \\
7,27 \\
10,9\end{array}$ & $\begin{array}{l}2,55 \\
2,16 \\
2,35 \\
3,15 \\
2,82 \\
1,88 \\
1,78 \\
2,05 \\
2,02 \\
2,06 \\
2,47 \\
3,27\end{array}$ & $\begin{array}{l}4,95 \\
4,59 \\
4,38 \\
5,85 \\
5,03 \\
4,01 \\
3,66 \\
4,28 \\
4,32 \\
3,85 \\
4,8 \\
7,63\end{array}$ \\
\hline $\begin{array}{l}\text { Oiseaux } \\
\text { Aiopochen aegyptiacus } \\
\text { Dendrocygna viduata } \\
\text { Irumida meleagris }\end{array}$ & $\begin{array}{l}4,88 \\
5,37 \\
5,1\end{array}$ & $\begin{array}{l}1,9 \\
1,89 \\
1,81\end{array}$ & $\begin{array}{c}2,9 \\
3,48 \\
3,29\end{array}$ \\
\hline
\end{tabular}


3. Méthode chimique d'estimation des protéines.

Par cette méthode, seules ont été déterminées quantitativement les matières protéiniques totales; les valeurs absolues des albumines ef des globulines totales ont été calculées à partir des valeurs relatives (tableau 6 ).

\section{CONCLUSION}

Ces premières investigations donnent un aperçu général de la distribution des constituants sériques chez les animaux sauvages du Centre africain, ef apportent une contribution à leur meilleure connaissance.

La détermination des limites normales ef l'étude de certaines variations plasmatiques caractéristiques peuvent être des indices pour le diagnostic et le pronostic de nombreux états pathologiques. Par voie de conséquence, elles permettent ainsi de préciser le rôle des animaux sauvages dans l'épizootologie de quelques-unes des maladies animales les plus importantes observées en Afrique.

\section{REMERCIEMENTS}

Nous tenons à associer dans nos remerciements, toutes les personnes qui ont participé à l'abattage des animaux et à la récolte des échantillons sanguins: Messieurs BORREDON, KERAVEC, LEVIF, PITON, PROVOST, SERVICE ef TYRAN.

\section{SUMMARY}

Electrophoretic study of serum proteins of Chad herbivora and birds. First results

The electrophoretic analysis of protidic components of the wild animals serum and of their relative mobility was carried out on 26 zoological genus including 119 mammals and 74 birds from Chad cygenetic fauna.

\section{RESUMEN}

Estudio electroforetico de las proteinas sericas de herbivoros $y$ de aves de la fauna de Chad. Primeros resultados

Se realizó el analisis electroforetico de los constituyentes protidicos del suero de animales salvajes $y$ de su movilidad relativa en 26 generos zoologicos incluyendo 119 mamíferos y 74 aves perteneciendo a la fauna cinegetica de Chad.

\section{BIBLIOGRAPHIE}

FINE (J.M.), GROULADE (J.), SAINT-PAUL (M.) et TIZZANI (R. J.). - L'électrophorèse sur papier. Biol. Méd., 1956, 45, no 6, 591-644.

DREVON (B.). - Intérêt d'une présentation normalisée des résultats de l'électrophorèse du sérum. Ann. Biol. Clin., 1956, 14, 66-70.

DREVON (B.). - Mobilités ef positions des protéines au cours de l'électrophorèse sur papier. Interprétation graphique des diagrammes d'électrophorèse. C. R. Soc. Biol., 1953, 147, 1416.

CHARY (R.) et DUFOUR (R.).- Recherches sur la constitution protéique des sérums animaux. Rev. Serv. Biol. et Vét., 1965, 18, no 3, 139.

CHARY (R.), DUFOUR (R.) et GAL (J. Y.). Recherche sur la constitution protéique des sérums animaux. Rev. Serv. Biol. Vét., 1966, 19, n० 2, 55 .

GORNALL (A. G.), BARDAWILL (C. J.) ef DAVID (M. M.). - Determination of serum proteins by means of the biuret reaction. J. Biol. chem., 1949, 177, 751-756.

FLEURY (D.) et COURTOIS (J. E.). - Fiches techniques de chimie biologique. Paris, Vega 1961). 